\title{
Systematic Study Multiplicity Production Nucleus - Nucleus Collisions at 4.5 a GeV/c
}

\author{
AAbd El-Daiem \\ Department of Physics, Faculty of Science, Sohag University, Sohag Egypt
}

\begin{abstract}
The correlations between the multiplicity distributions and the projectile fragments, as well as the correlation between the black and grey fragments were given. We observed that the mean number of interacting projectile nucleons increases quickly as the value of heavily ionizing charged particles increase as expected but attains a more or less constant value for extreme central collisions. Finally, there is no distinct correlation between the shower particle production and the target excitation, but the average value of grey particles decreases with the increase of the number of black particles and vice versa. This correlation can also be explained by the fireball model.
\end{abstract}

Keywords: Correlations between the multiplicity distributions, Total disintegrations.

\section{Introduction}

The interest in high energy nucleus-nucleus collisions has a greatly importance during the few recent years. It allows one to obtain some information about the mechanisms of the multiparticle production and the structure of hot nuclear matter [1]. From the participant spectator model [2], the quark gluon plasma (quark matter) will be formed in the participant between the projectile and the target at very high incident energies and a liquid gas phase transition will occur in the spectator [3].The interaction between the incident beam and the target emulsion is called central when the impact parameter is less than the absolute value of the difference between the interacting radii of two nuclei, i.e. $\mathrm{b}<1 \mathrm{Rp}$ - Rtl where Rpand Rtare the projectile and target nuclei radii respectively. Such events are characterized by large multiplicities of the generated particles and the emitted target fragments. The emission of particles is symmetric with respect to the direction of the incident beam. In central collision all projectile nucleons are participants and no projectile spectators. The study of the correlation in complete destruction of $\mathrm{Ag}(\mathrm{Br})$ emulsion nuclei is very interesting. The most of these interactions are due to central collision. The central collision provides a unique opportunity to investigate the consequents of nuclear compression, such as hydrodynamic effects [4-6]. The aim of the present research is to perform a systematic analysis of target fragmentation in ${ }^{24} \mathrm{Mg}$ and ${ }^{28} \mathrm{Si}$ with emulsion at $4.5 \mathrm{~A} \mathrm{GeV} / \mathrm{c}$. The second section describes the experimental materials. The correlations between the multiplicity distributions and the projectile fragments are given in the third. Multiplicity of charged particles and total disintegration events of heavy emulsion targets caused by ${ }^{28} \mathrm{Si}$ at $4.5 \mathrm{~A} \mathrm{GeV} / \mathrm{c}$, fourth and fifth sections respectively. The last section gives our conclusion.

\section{Experimental Details}

The present research was carried out using stacks of $\mathrm{Br}-2$ nuclear emulsions exposed to $4.5 \mathrm{~A} \mathrm{GeV/c}$ ${ }^{24} \mathrm{Mg}$ and ${ }^{28} \mathrm{Si}$ beams at the DubnaSynchrophastorn. The stacks have dimensions of $20 \mathrm{cmx} 10 \mathrm{~cm}$ x $600 \mu \mathrm{m}($ undeveloped emulsions). The intensity of irradiation was $\sim 10^{4}$ particle $\mathrm{cm}^{-2}$ and the beam diameter was about 1 $\mathrm{cm}$. Along the track double scanning was carried out fast in the forward and slow in the backward direction. The scanned beam tracks were further examined by measuring the delta electron density on each of them to exclude the tracks having charge less than the beam particle charge $\mathrm{Z}_{\mathrm{b}}$. According to the range $\mathrm{R}$ in the emulsion and the relative ionization $I^{*}=I / I_{0}$ (where $I$ is the particle track ionization and $I_{0}$ is the ionization of relativistic shower tracks in the narrow forwards cone of an opening angle of $\theta \leq 3^{\circ}$ ) all charged secondary particles in the found interactions were classified into the following groups. (i) The shower tracks correspond to relativistic charged particles, whereas grey and black tracks are produced by relatively slow particles emitted from the target nuclei. (ii) Grey tracks are mostly recoil protons with $40<\mathrm{E}>400 \mathrm{MeV}$, and range $\mathrm{R}>3 \mathrm{~mm}$ in emulsion with less than a few percent admixture of low energy pions. (iii) Black tracks are due slow particles and evaporated target fragments with $\mathrm{E}<40 \mathrm{~A} \mathrm{MeV}$, and range $\mathrm{R} \leq 3 \mathrm{~mm}$. The sum of grey and black tracks in an interaction is represented by $\mathrm{N}_{\mathrm{h}}\left(=\mathrm{N}_{\mathrm{g}}+\mathrm{N}_{\mathrm{b}}\right)$, and these tracks are thought to originate from heavily ionizing charged particles. In each interaction we have taken the following measurement. The total charge of the projectile fragments $Q=$ $\sum n_{i} z_{i}$ where $n_{i}$ is the number of fragments of charge $z_{i}$ in an even that of the given tracks.

\section{Results and discussion \\ 3.1. Correlations between Multiplicity Distribution and Projectile Fragments}

The charges of the projectile fragments can be measured in a nuclear emulation by the grain density and delta-ray counting methods [7] as well as the lacunarity technique [8]. Thecharge identification of 
relativistic fragment (of $Z \geq 2$ ) has been made by measuring the gap length along the track which is associated with the energy loss. To identify the charge of a trackby this method, one measures the frequency of the gaps with a length $\geq 2 \mu \mathrm{m}$ in a distance of $2 \mathrm{~cm}$ along the track starting from the vertex. Thus, by counting the number of gaps of two tracks and knowing the charge of one, then the charge of the other can be determined from the inverse proportionality of gaps with charges. In this research, the charges of the projectile spectator were identified by means of the delta ray counting method. Let $\mathrm{n}_{\mathrm{Mg}}, \mathrm{n}_{\mathrm{Si}}$ and $\mathrm{n}_{\mathrm{z}}$ denote the experimental track delta ray densities of the incident ${ }^{24} \mathrm{Mg},{ }^{28} \mathrm{Si}$ nucleus and projectile fragment with charge respectively. In nuclear emulsion we can measure the charges of all projectile fragments. Then the number of interacting nucleons of the projectilenucleus is on average $\mathrm{N}_{\mathrm{int}}=\mathrm{A}_{\mathrm{p}}-2 \mathrm{Q}$, where $\mathrm{Q}$ is the total charge. The relationship between $\mathrm{N}_{\mathrm{i}}(\mathrm{i}=\mathrm{g}, \mathrm{b}$, h) with Q should be observed [9]. Figure 1 shows the correlations between $\mathrm{N}_{\mathrm{i}}$ and $\mathrm{Q}$ at $4.5 \mathrm{~A} \mathrm{GeV/c}$ (open circles) for ${ }^{24} \mathrm{Mg}$ and (solid circles) for ${ }^{28} \mathrm{Si}$ with emulsion collision. Figure $1 \mathrm{a}, \mathrm{b}$ and $\mathrm{c}$ correspond to the correlation $\left\langle\mathrm{N}_{\mathrm{i}}\right\rangle$ with Q respectively. One can see that negative correlations between $\langle\mathrm{Ni}\rangle$ and $\mathrm{Q}$ are obtained. The $\mathrm{N}_{\mathrm{i}}$ distributions for events with different $\mathrm{Q}$ regions are shown in Fig. 2, 3 and 4receptively. The dotted and solid histogram correspond to the experimental data for ${ }^{24} \mathrm{Mg}$ and ${ }^{28} \mathrm{Si}$ with emulsion collisions at $4.5 \mathrm{~A} \mathrm{GeV} / \mathrm{c}$ Fig. $1 \mathrm{a}, \mathrm{b}$ and $\mathrm{c}$ are the results for events with $\mathrm{Q}=0, \mathrm{Q}=2-6$ and $\mathrm{Q}=7-12$ for ${ }^{24} \mathrm{Mg}$ and $\mathrm{Q}=0, \mathrm{Q}=2-7$ and $\mathrm{Q}=8-14$ for ${ }^{28} \mathrm{Si}$. One can see that themultiplicity of fragments has a wide and even distribution at small $\mathrm{Q}$. The number of events with low multiplicity increases and the number of events with high multiplicity decreases with increasing value of $\mathrm{Q}$. The multiplicity distribution becomes narrow at great $\mathrm{Q}$. The mean multiplicities of events with different $Q$ are shown in Table 1. One can see that the mean multiplicities decrease with increasing value of $\mathrm{Q}$. The negative correlation between $\mathrm{N}_{\mathrm{i}}$ and $\mathrm{Q}$ is determined by the nuclear geometry. For a projectile and target, $\mathrm{N}_{\mathrm{i}}$ increases with decreasing the impact parameter. For $\mathrm{N}_{\mathrm{b}}$, there is a saturation effect appearing if the impact parameter is small enough i.e., $\mathrm{N}_{\mathrm{b}}$ does not decrease. But the projectile spectator, the value of $\mathrm{Q}$ decreases with increasing the impact parameter.

\subsection{Multiplicity of Secondary Particles}

The Multiplicity characteristics provide a valuable tool to investigate the mechanism of the nucleusnucleus interaction. The study of the secondary track multiplicities (shower, grey and black particles) from heavy ion interactions provides information about the mechanism of production of these particles. At high energy the nuclear interaction is a collision between the incident projectile and the nucleon of the target. The energy transfer during these collisions creates relativistic particles. In the present paper, the dependence of the secondary particles on the number of heavily ionizing tracks Nhhas been studied. It is known that the impact parameter is difficult to be measured experimentally, so the multiplicity of the target fragment Nbconsidered as a measure of the impact parameter (11): the higher the $\mathrm{Nh}$, the lower the impact parameter, and vice versa. The average multiplicities $\langle\mathrm{NS}\rangle,\langle\mathrm{Ng}\rangle,\langle\mathrm{Nb}\rangle,\langle\mathrm{Nh}\rangle$ and $\langle\mathrm{Nint}\rangle$ for ${ }^{24} \mathrm{Mg}$ and ${ }^{28} \mathrm{Si}$ interactions at different impact parameter are presented in Table 2. It is clear that these values increase as Nhincreases (decreasing impact parameter). The highest value of them is obtained for events. With $\mathrm{Nh} \geq 28$ for which complete destruction of the heavy component $\mathrm{AgBr}$ of the target nuclei takes place. From Table2, it can be seen that the average multiplicities of the secondary particles depend on the impact parameter. This also means that the degree of centrality of the interaction increases at smaller impact parameters. Figure 5 represents the experimental data of the probability distributions of shower, gray, black and compound multiplicity particles for the interaction of ${ }^{24} \mathrm{Mg}$ and ${ }^{28} \mathrm{Si}$ with emulsion. From this figure, one can see that the $\mathrm{Nb}$ multiplicity distribution ${ }^{28}{ }^{28} \mathrm{Si}$ with emulsion is almost similar to that in ${ }^{24} \mathrm{Mg}$ with emulsion interactions, which indicate that the target-associated particles are characterized by a weak dependence on the projectile mass number Ap. This result is consistent with [10,11]. The Ns and Ng multiplicity distributions become broader with the increase of Ap. The highest value of the multiplicity in Figure 5, accurse at small values of Ns and decreases as Apof the projectile increases. As already known, the grey particles are emitted shortly after the passage of the leading hadron. Therefore, one may expect that it is worth to use the compound multiplicity of the grey and shower particles. The variable $\mathrm{Nc}$ which equals the sum of $\mathrm{Ns}$ and $\mathrm{Ng}$ is used to study the particle production mechanism. The compound multiplicity for ${ }^{24} \mathrm{Mg}$ and ${ }^{28} \mathrm{Si}$ at $4.5 \mathrm{~A} \mathrm{GeV} / \mathrm{c}$ interactions for these two reactions are consistent with each other, and it can be seen that the peak of the distribution shifts towards a higher value of Nc with decreasing projectile mass. Also, it may further be seen that the Nc- distributions becomes border with increasing projectile mass.

\subsection{Total Disintegration Events of Heavy Emulsion Targets Produced by $4.5 \mathrm{AGeV} / \mathrm{c}$.}

In this section, the criterion $\mathrm{Nh} \geq 28$ is used to select events for the total disintegration of $\mathrm{AgBr}$ nuclei in nuclear emulsion. It is found that this condition corresponds to the destruction of target nucleus into nearly individual nucleons without a measurable residual nucleus existing. Since then; this criterion is used to select the total disintegration events of $\mathrm{AgBr}$. The multiplicity correlations among various charged secondary produced in nucleus-nucleus collision have been studied exclusively. However, little attention has been paid to studying the multiplicity correlations of these secondary charged particles in the total disintegration of $\mathrm{AgBr}$ nuclei caused by 
the projectile. Thus, in this work, we have studied some multiplicity correlations of the type $\langle\mathrm{Ni}(\mathrm{Nj})\rangle$, where $\mathrm{i}$ and $\mathrm{j}$ mean shower, grey and black particles, and $\mathrm{i} \neq \mathrm{j}$ for the total disintegration events produced in ${ }^{28} \mathrm{Si}$ with $\mathrm{AgBr}$ interactions at $4.5 \mathrm{~A} \mathrm{Gev} / \mathrm{c}$. From Fig. 6 we can see that there are practically no dependences of and $\langle\mathrm{Ng}\rangle$, $\langle\mathrm{Nb}\rangle$ on $\mathrm{Ns}$ for the total disintegration events of ${ }^{28} \mathrm{Si}$ with $\mathrm{AgBr}$ collision, signifying that the target excitation is independent of the production of relativistic particles in the total disintegration events, which means that the target excitation reach its maximum in the total disintegration events. From Figs 7 and 8 we can also see that there are prectically no dependences of $\left\langle\mathrm{N}_{\mathrm{s}}\right\rangle$ on $\mathrm{N}_{\mathrm{g}}$ and $\mathrm{N}_{\mathrm{b}}$, signifying that the number of relativistic particles produced in total disintegration events is independent of the target excitation. The dependence of $\langle\mathrm{Nb}\rangle$ on $\mathrm{Ng}$ is plotted in Fig.7. It is clear from this figure that the value of $\left\langle\mathrm{N}_{\mathrm{b}}\right\rangle$ decreases with the value of $\mathrm{Ng}$ increasing, and vice versa, the value of $\langle\mathrm{Ng}\rangle$ decreases with the increase of $\mathrm{Nb}$ (see Fig.8). These observations further support our conclusion above and can be explained by the fireball model [12]. The correlation of $\left\langle\mathrm{N}_{\mathrm{b}}\right\rangle$ with $\mathrm{Ng}$ can be fitted by a linear relation in the form $\left\langle\mathrm{N}_{\mathrm{b}}\right\rangle=\mathrm{a} \pm \mathrm{b}$ Ng with best fitting parameter $\mathrm{a}=20.83 \pm 0.73$ and $\mathrm{b}=-0.35 \pm$ 0.03 . For the linear relation of $\langle\mathrm{Ng}\rangle$ to $\mathrm{Nb}$, the best fitting parameters are $\mathrm{a}=27.92 \pm 1.12$ and $b=-0.56 \pm 0.08$. It is interesting to note that all correlation coefficients of inclination in the total disintegration events of ${ }^{28} \mathrm{Si}$ with $\mathrm{AgBr}$ collision are negative, which are the same as the results from, $\mathrm{p}$-emulsion, central ${ }^{12} \mathrm{C}$ with $\mathrm{AgBr}$ collision at the same energy [13] and ${ }^{16} \mathrm{O}$ with $\mathrm{AgBr}$ collisions at the same energy [14].

\section{Conclusions}

From the investigation of particles emitted from ${ }^{24} \mathrm{Mg}$ and ${ }^{28} \mathrm{Si}$ with emulsion collisions, we can make the following conclusion: (i) Correlation between multiplicity distributions and projectile fragments, (ii) we can see that negative correlations between $\left\langle\mathrm{N}_{\mathrm{i}}\right\rangle$ and $\mathrm{Q}$, the number of events with high multiplicity decreases with increasing value of $\mathrm{Q}$ and themultiplicity distribution becomes narrow at great $\mathrm{Q}$.We observed that the mean number of interacting projectile nucleons increases quickly as the value of Nhincrease, as expected but attains a more or less constant value for extreme central collisions. (iii) There is no distinct correlation between the shower particle production and the target excitation, but the average value of grey particles decreases with the increase of the number of black particles, and vice versa. This correlation can also by explained by the fireball model.

[1]. OyniecGInt. J. Mod. Phys. A17 3107 (2002).

\section{Reference}

[2]. GlauberR G In Lectures of Theoretical Physics, (Eds) WE Britin, Dunham LG PhD dissertation Interscience, NewYourk 1: 315 (1959).

[3]. Mu Y GPhys. Lett.83 3617 (1997)

[4]. Beavis D S, Fung S Y, Gom W, Keano D, Y M Liu Y M, Poe R T, Van Dalen G and Vient MPhys.Rev. Lett.54 1652 (1979).

[5]. Beckmann P, Gustafasson H A, and Gutbrod H H, Kalmpert K H, Kolb B, Loehren H, Posanzer A M, Ritter H G,Shmidt H R, and Siemiarcz T CK, Mod. Phys.Lett.A2. 163- 168 (1987).

[6]. Csernai L P, Freier P, Mevissen J, Nguyen H, and Waters L, Phys.Rev.C 34, 1270(1986)

[7]. Jain P L, Aggarwal M M and Gomber K LPhys. Rev. C 34726 (1986)

[8]. Ghosh D, Das S K and Ghosh KNuovoCimento A110 565 (1997).

[9]. Solite M E, Abd El Daiem A, Int. J. Phys. Sci.Vol.2 (12) P 324-330(2007)

[10]. Tariq M et al Int. J. Mod.Phys. E4 347 (1995).

[11]. Sherif M M et al Int. J. Mod. Phys.E2 835 (1993).

[12]. Jilany M A, Nucl.Phys.A 579 627(1994).

[13]. Singh P, Khan M S and Khushnood HCan. J. Phys.76 559 (1998).

[14]. Zhang D H et al Chin.Phys.Vol.15 No 11 November (2006)

Table 1: Average multiplicities of target fragments in ${ }^{24} \mathrm{Mg}$ and ${ }^{28} \mathrm{Si}$ with emulsion collisions at $4.5 \mathrm{~A} \mathrm{GeV} / \mathrm{c}$.

\begin{tabular}{|r|r|r|r|r|}
\hline Projectile & $\mathrm{Q}$ (region) & $\left\langle\mathrm{N}_{\mathrm{g}\rangle}\right.$ & $\left\langle\mathrm{N}_{\mathrm{b}\rangle}\right.$ & $\left\langle\mathrm{N}_{\mathrm{h}\rangle}\right.$ \\
\hline${ }^{24} \mathrm{Mg}$ & $\mathrm{Q}=0$ & $22.1 \pm 1.1$ & $10.1 \pm 1.1$ & $32.1 \pm 1.2$ \\
\cline { 2 - 5 } & $\mathrm{Q}=2-6$ & $8.8 \pm 0.9$ & $6.20 \pm 0.7$ & $14.8 \pm 0.9$ \\
\cline { 2 - 5 } & $\mathrm{Q}=7-12$ & $2.2 \pm 0.4$ & $2.30 \pm 0.5$ & $2.40 \pm 0.3$ \\
\hline \multirow{3}{*}{${ }^{28} \mathrm{Si}$} & & $19.4 \pm 0.8$ & $11.9 \pm 1.5$ & $31.3 \pm 1.0$ \\
\cline { 2 - 5 } & $\mathrm{Q}=0$ & $10.8 \pm 0.9$ & $7.90 \pm 1.3$ & $18.8 \pm 1.2$ \\
\cline { 2 - 5 } & $\mathrm{Q}=2-7$ & $2.40 \pm 0.3$ & $2.80 \pm 0.3$ & $5.50 \pm 0.7$ \\
\hline
\end{tabular}


Table 2. Average secondary charged particles multiplicities of inelastic interactions of ${ }^{24} \mathrm{Mg}$ and ${ }^{28} \mathrm{Si}$ with emulsion at $4.5 \mathrm{~A} \mathrm{GeV/c}$.

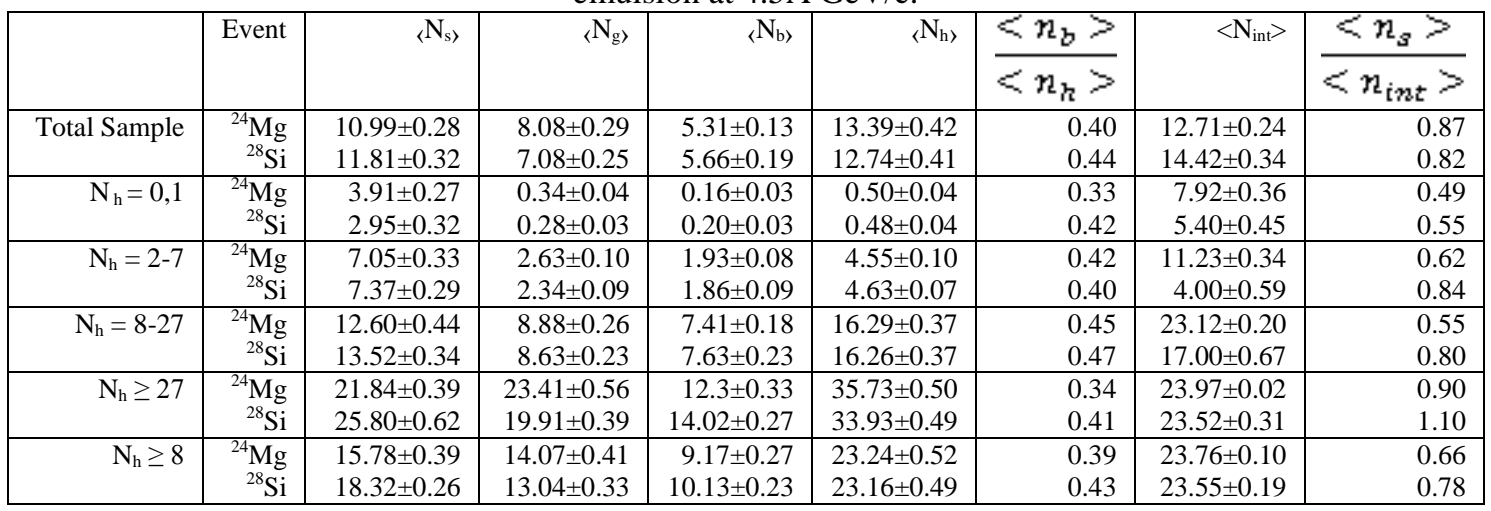

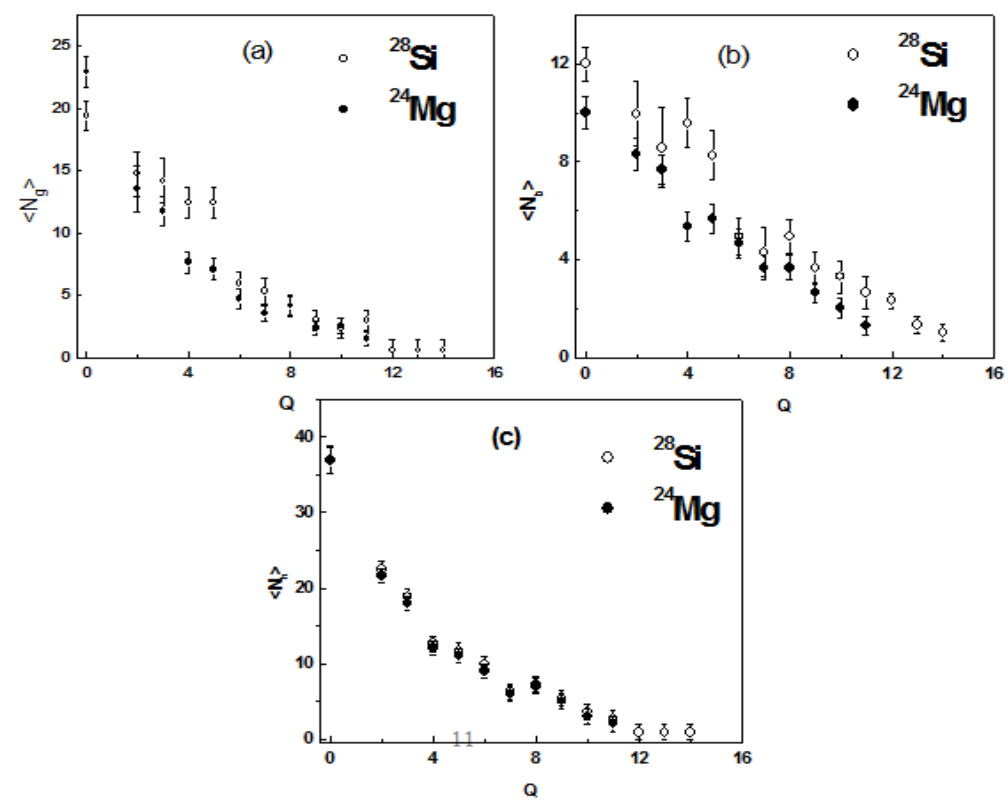

Fig.1 (a) Correlation between $\left\langle\mathrm{N}_{\mathrm{g}}\right\rangle$ and $\mathrm{Q}$, (b) Correlation between $\left\langle\mathrm{N}_{\mathrm{b}}\right\rangle$ and $\mathrm{Q}$, (c) Correlation between $\left\langle\mathrm{N}_{\mathrm{h}}\right\rangle$ and $\mathrm{Q}$, in ${ }^{24} \mathrm{Mg}$ and ${ }^{28} \mathrm{Si}$ interactions with emulsion at $4.5 \mathrm{~A} \mathrm{GeV} / \mathrm{c}$.

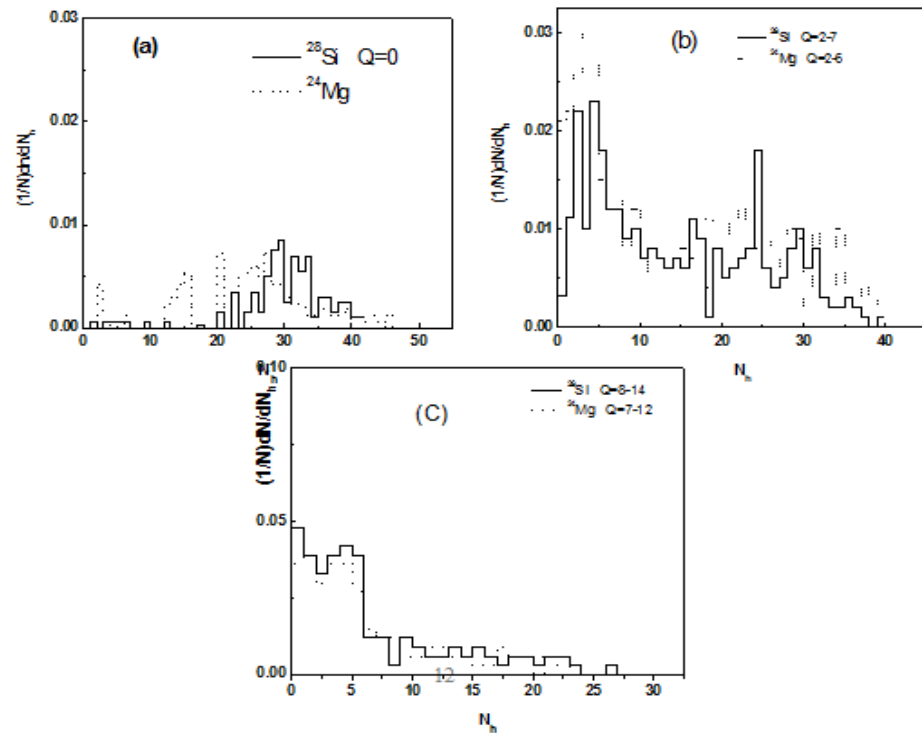

Fig. 2 (a) The $\mathrm{N}_{h}$ distributions for events with $\mathrm{Q}=0$, (b) The $\mathrm{N}_{\mathrm{h}}$ distribution for events with $\mathrm{Q}=2-6,2-7$, (c) The $\mathrm{N}_{\mathrm{h}}$ distribution for events with $\mathrm{Q}=7-12,8-14$, in ${ }^{24} \mathrm{Mg}$ and ${ }^{28} \mathrm{Si}$ interactions with emulsion at $4.5 \mathrm{~A} \mathrm{GeV} / \mathrm{c}$ 

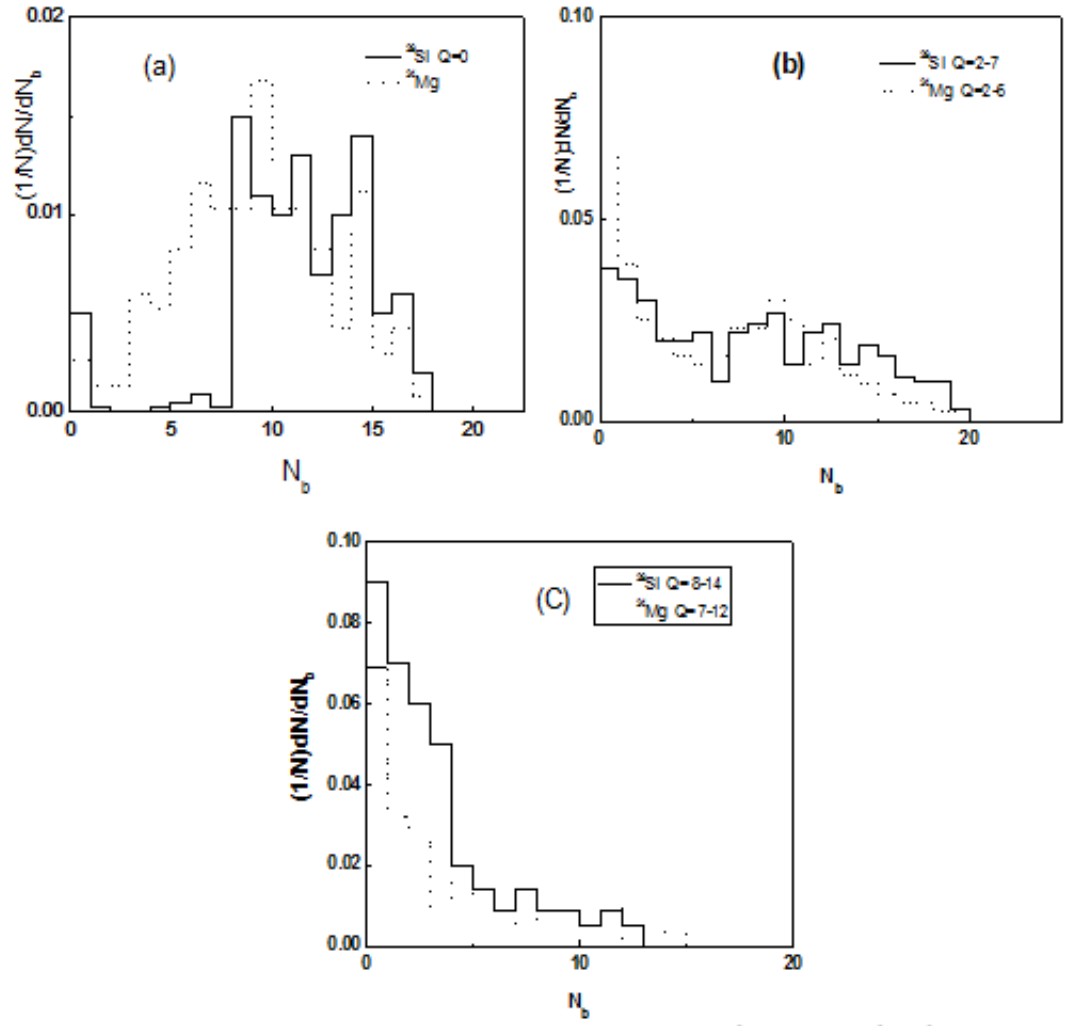

Fig. 3 (a) The $\mathrm{N}_{\mathrm{b}}$ distributions for events with $\mathrm{Q}=0$, (b) The $\mathrm{N}_{\mathrm{b}}$ distribution for events with $\mathrm{Q}=2-6,2-7$, (c) The $\mathrm{N}_{\mathrm{b}}$ distribution for events with $\mathrm{Q}=7-12,8-14$, in ${ }^{24} \mathrm{Mg}$ and ${ }^{28} \mathrm{Si}$ interactions with emulsion at $4.5 \mathrm{~A} \mathrm{GeV} / \mathrm{c}$
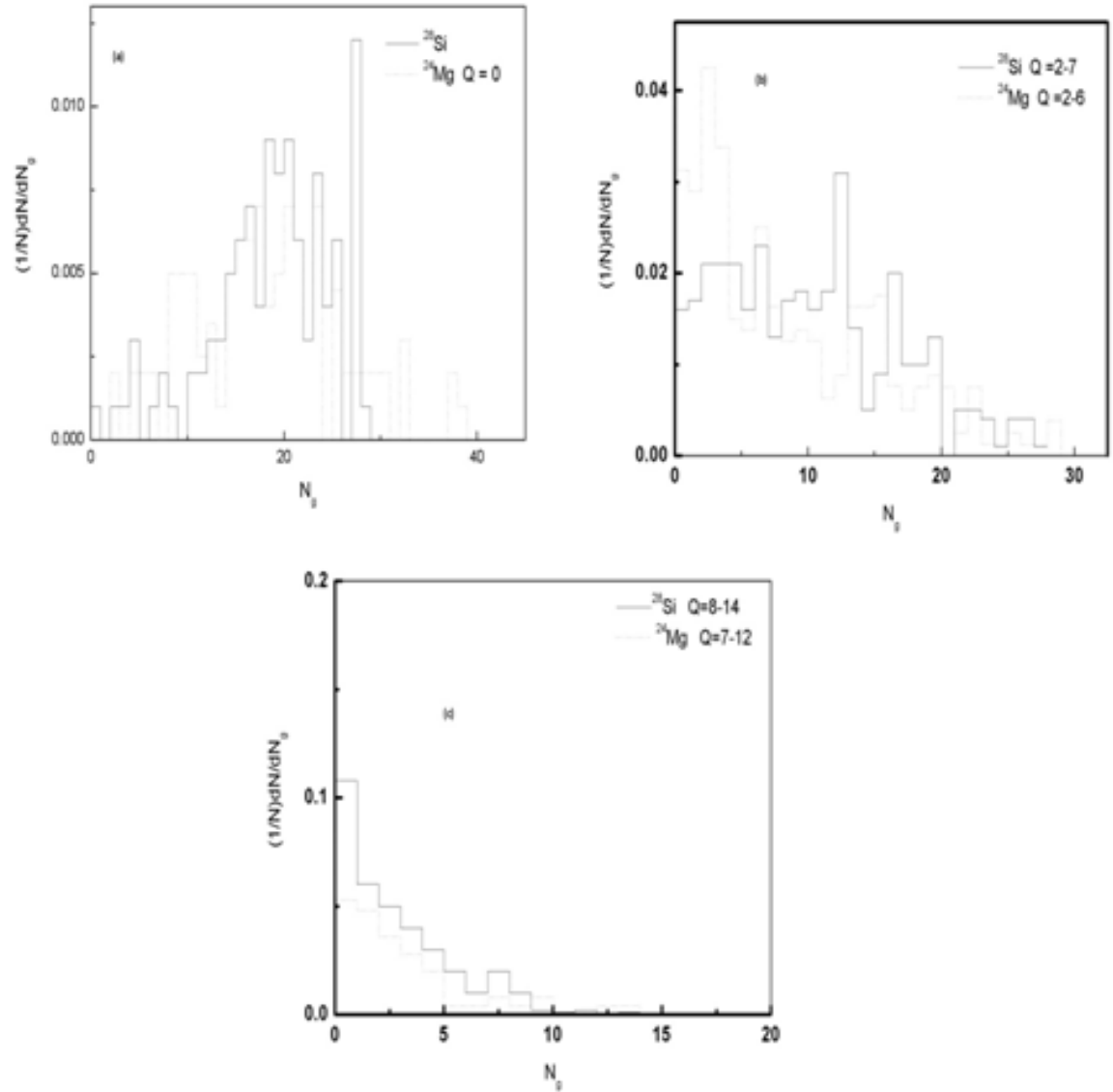

Fig. 4 (a) The $\mathrm{N}_{\mathrm{g}}$ distributions for events with $\mathrm{Q}=0$, (b) The $\mathrm{N}_{\mathrm{g}}$ distribution for events with $\mathrm{Q}=2-6,2-7$, (c) The $\mathrm{N}_{\mathrm{g}}$ distribution for events with $\mathrm{Q}=7-12,8-14$, in ${ }^{24} \mathrm{Mg}$ and ${ }^{28} \mathrm{Si}$ interactions with emulsion at $4.5 \mathrm{~A} \mathrm{GeV} / \mathrm{c}$ 

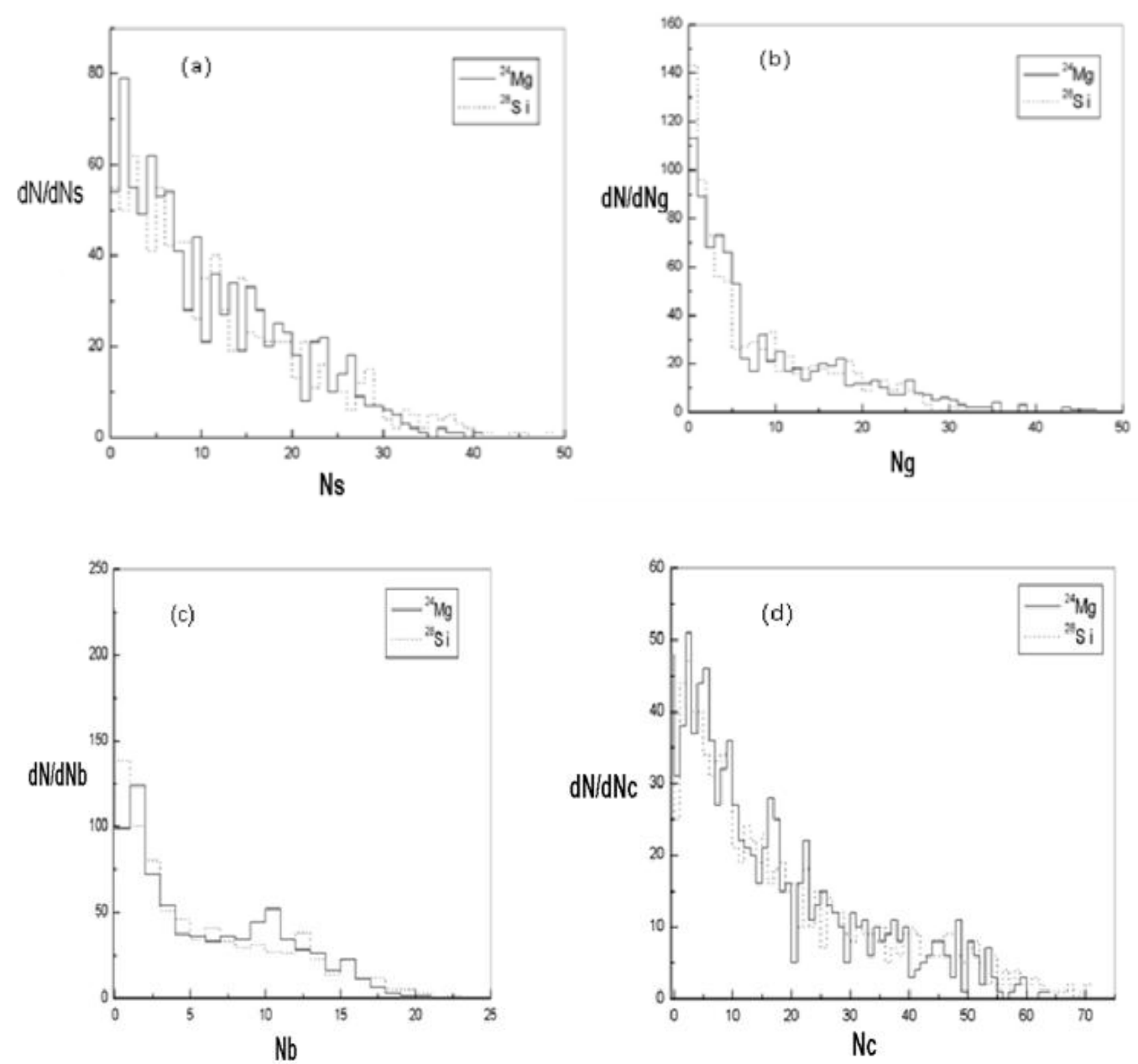

Fig. 5 (a) Multiplicity distribution of shower $\mathrm{N}_{\mathrm{s}}$, (b) Multiplicity distribution of gray $\mathrm{N}_{\mathrm{g}}$, (c) Multiplicity distribution of black $\mathrm{N}_{\mathrm{b}}$ and (d) Multiplicity distribution of compound particles $\mathrm{N}_{\mathrm{c}}$, emitted from the ${ }^{24} \mathrm{Mg}$ and ${ }^{28} \mathrm{Si}$ interactions with emulsion at $4.5 \mathrm{~A} \mathrm{GeV} / \mathrm{c}$.

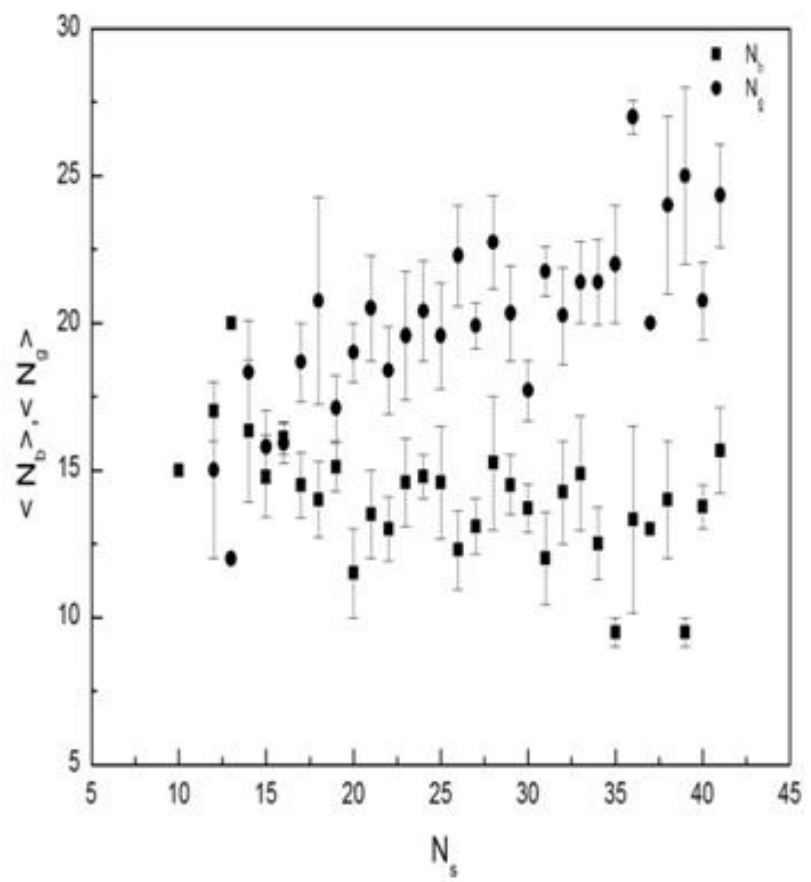

Fig. 6 Variations of $\left\langle\mathrm{N}_{\mathrm{g}}\right\rangle$ and $\left\langle\mathrm{N}_{\mathrm{b}}\right\rangle$ as a function of $\mathrm{N}_{\mathrm{s}}$. 


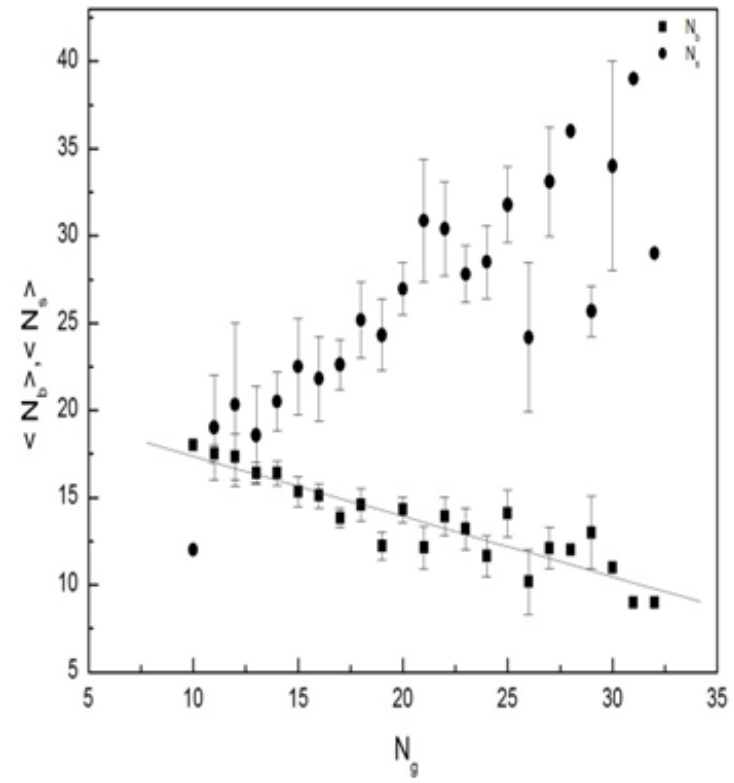

Fig.7 Dependence $\left\langle\mathrm{N}_{\mathrm{s}}\right\rangle$ and $\left\langle\mathrm{N}_{\mathrm{b}}\right\rangle$ on $\mathrm{N}_{\mathrm{g}}$.

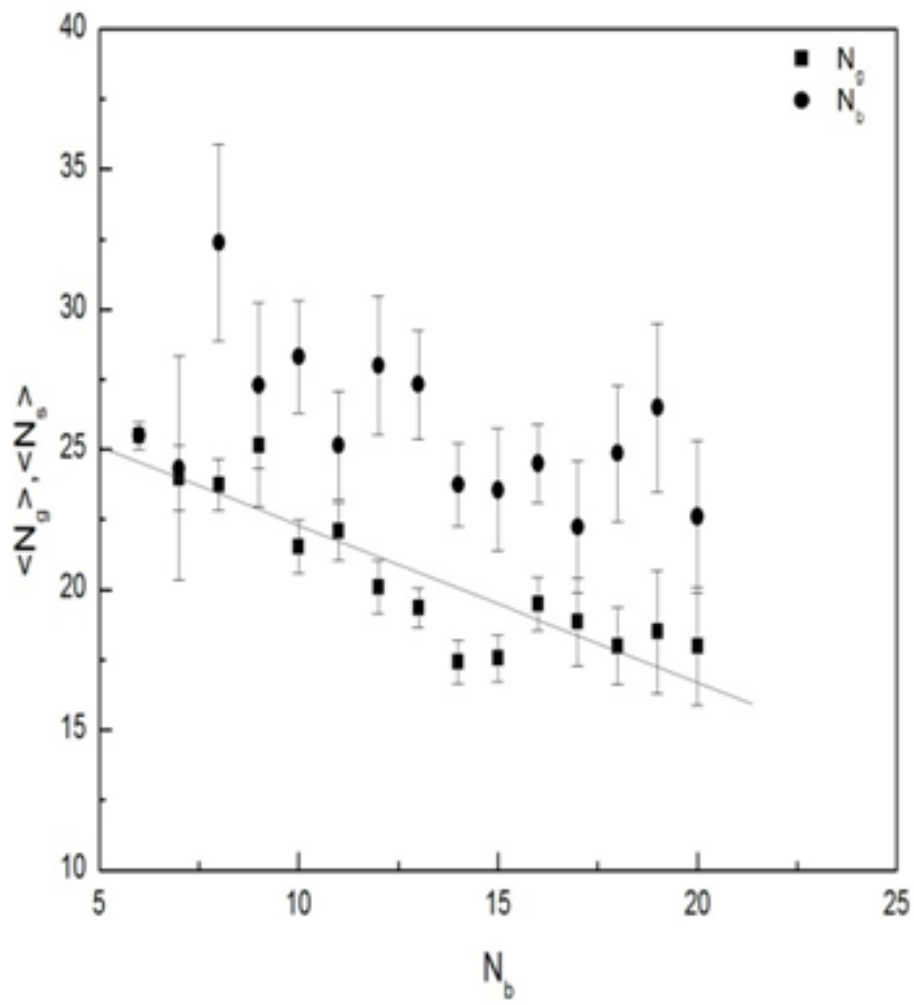

Fig. 8 Dependence $\left\langle\mathrm{N}_{\mathrm{s}}\right\rangle$ and $\left\langle\mathrm{N}_{\mathrm{g}}\right\rangle$ on $\mathrm{N}_{\mathrm{b}}$. 